\title{
Vector averaging of inhibition of return
}

\author{
RAYMOND M. KLEIN, JOHN CHRISTIE, and ERIC P. MORRIS \\ Dalhousie University, Halifax, Nova Scotia, Canada
}

\begin{abstract}
Observers detected targets presented $400 \mathrm{msec}$ after a display containing one cue or two to four cues displayed simultaneously in randomly selected locations on a virtual circle around fixation. The cue arrangement was completely uninformative about the upcoming target's location, and eye position was monitored to ensure that the participants maintained fixation between the cue and their manual detection response. Reflecting inhibition of return (IOR), there was a gradient of performance following single cues, with reaction time decreasing monotonically as the target's angular distance from the cued direction increased. An equivalent gradient of IOR was found following multiple cues whose center of gravity fell outside the parafoveal region and, thus, whose net vector would activate an orienting response. Moreover, on these trials, whether or not the targeted location had been stimulated by a cue had little effect on this gradient. Finally, when the array of cues was balanced so that its center of gravity was at fixation, there was no IOR. These findings, which suggest that IOR is an aftermath of orienting elicited by the cue, are compatible with population coding of the entire cue (as a grouped array for multiple cues) as the generator of IOR.
\end{abstract}

Shortly after presentation of an uninformative peripheral cue, performance is usually enhanced at the cued location, relative to the opposite, uncued location, an effect that has been attributed to the capture of attention by the cue. Posner and Cohen (1984) demonstrated that after attention is withdrawn from the cued location, the pattern reverses, and responses to cued targets are delayed. This deficit has been called inhibition of return (IOR), to reflect the hypothesis that attention, having recently been removed from a peripherally cued location, was subsequently inhibited from returning there. IOR has received considerable attention since its discovery (see Klein, 2000, for a review) - in part, because of its proposed function of encouraging orienting to novelty (Posner \& Cohen, 1984), which in turn might facilitate foraging activities such as visual search (Klein, 1988). Whereas this research activity has produced fairly consistent findings, leading to general (although far from universal) agreement on several key properties, there are some findings that stand out, challenging conventional wisdom about some of these properties. In this article, we explore one such finding, which has implications for ideas about the cause of IOR and its spatial distribution.

How is IOR distributed when multiple locations are simultaneously cued? ${ }^{1}$ Wright and Richard (1996) sought an answer by presenting one cue or two, three, or four cues simultaneously at any of eight locations on a circle surrounding fixation. Two methods that Posner and Cohen (1984) had used to ensure that attention might not remain at the cue location(s) were adopted: Many (one third) of the targets were presented at fixation, and a return cue was presented at fixation after the cue display and im-

Correspondence concerning this article should be addressed to R. M. Klein, Department of Psychology, Dalhousie University, Halifax, NS, B3H 4J1 Canada (e-mail: ray.klein@dal.ca). mediately before the target's appearance. The average reaction time (RT) to cued targets minus the average RT to uncued targets was roughly the same regardless of the number of cues, leading Wright and Richard (1996) to conclude that "IOR occurred with equal magnitude at up to four locations at the same time" (p. 326).

This conclusion about how IOR is distributed following multiple simultaneous cues has a bearing on a longstanding question in the IOR literature: Is IOR generated by local stimulation (Posner \& Cohen, 1984), or is it an aftermath of the activation of the machinery responsible for orienting - in particular, overt orienting (Maylor, 1985)? Wright and Richard's (1996) claim of equal amounts of IOR at up to four randomly positioned locations seems to reinforce the local stimulation proposal, and because an eye movement can be made to only one location at a time, it also challenges the widely held view (Klein, 2000; Rafal, Calabresi, Brennan, \& Sciolto, 1989; Taylor \& Klein, $1998)$ that IOR is generated by oculomotor programming.

Several aspects of Wright and Richard's (1996) study, however, warrant caution when one interprets their findings. First, their cue display predicted the target's location in a way that was confounded with (indeed, inversely related to) the number of cued locations. The number of cued and uncued trials was the same regardless of the number of cued locations. With eight possible target locations and a four-cue array, there was an equal probability of a peripheral target's appearing in each of the eight locations, and thus the four-element cue was uninformative. With one cued location, because one half of the peripheral targets were presented at the cued location, whereas the remaining one half were presented in one of the remaining seven locations, a peripheral target was seven times more likely to appear at the cued than at an uncued location. Trials with two and three cued locations 
entailed intermediate levels of cue informativeness. When a cue predicts the target's location, conventional wisdom suggests that attention might be maintained at the cued location, and then IOR might not be generated, or it might be overshadowed by attentionally mediated facilitation (Klein, 2000). Confirming this "wisdom," Wright and Richard (2000) found that "the inhibition-of-return effect occurred only when the cue provided no information about the impending target's location" (p. 2351). The cued RTs in Wright and Richard (1996) are likely contaminated by indeterminate amounts of attentional facilitation, making them suspect as a measure of IOR. Second, Wright and Richard's (1996) operational definition of IOR (average cued minus average uncued RT) is too crude to support claims about the spatial distribution of inhibition following cue displays with one to four cues. Following a single cue, for example, collapsing uncued performance across different cue-target distances (ranging from adjacent to opposite) ignores the likelihood that there is a gradient of inhibition (Bennett \& Pratt, 2001; Dorris, Taylor, Klein, \& Munoz, 1999; Pratt, Spalek, \& Bradshaw, 1999, Experiment 3) that decreases monotonically as the distance between the cue and the target increases. Moreover, even though Wright and Richard (1996) rejected oculomotor mechanisms as the IOR generator, "because eye movements can be programmed and executed to only one location at a time" (p. 326), their data were not analyzed directly with respect to the underlying hypothesis that eye movements might be programmed in one direction at a time.

Here, we repeated Wright and Richard's (1996) multiple simultaneous cuing procedure, but making the following improvements: (1) The number of peripheral cues was varied while, simultaneously, the cues were kept completely uninformative about the upcoming target's location; (2) performance as a function of the relationship between the net direction of the cue and the target's direction in relation to fixation was considered, in addition to whether the target appeared at a cued versus an uncued location; and (3) eye position was monitored so that trials with shifts of gaze prior to the detection of the target could be excluded from analysis. Implementing these features, we discovered a robust inhibitory gradient centered in the direction of the net vector of the cue that had the same magnitude, whether one or more cues had been presented and whether or not the target fell on a cued, as opposed to an uncued, location. This pattern of results strongly implicates orienting in response to the cue array as a critical factor underlying the generation of IOR.

\section{METHOD}

\section{Participants}

Twenty-two Dalhousie University psychology students volunteered in the study and received course credit. All the participants had either normal or corrected-to-normal vision.

\section{Apparatus and Stimuli}

All the stimuli were presented on a 17 -in. SVGA monitor controlled by a computer with an AMD Duron 600 processor. A video- based EyeLink system was used to monitor the participants' eye positions from the initiation of the trial until the manual detection response. The button box response apparatus, EyeLink headset, and display computer were connected to a second computer with a Pentium 166 processor. After a 9-point calibration, this computer provided accurate information about gaze position every $4 \mathrm{msec}$.

The cues and targets were presented in white at fixation and at eight equally spaced locations on an imaginary circle with a radius of $7.6^{\circ}$, centered on an unlit black screen that was viewed from a distance of approximately $26 \mathrm{in}$. The initial fixation stimulus was a white cross $\left(0.4^{\circ} \times 0.4^{\circ}\right)$, located at the center of the imaginary circle, and remained on the screen for the entire trial. The cues were unfilled white squares $\left(1.9^{\circ} \times 1.9^{\circ}\right)$. The target consisted of a solid white square $\left(0.95^{\circ} \times 0.95^{\circ}\right)$. See Figure 1 for some sample displays.

\section{Procedure}

The participant was instructed to fixate the white fixation cross for the entire trial, was told that the cue(s) did not predict the up-

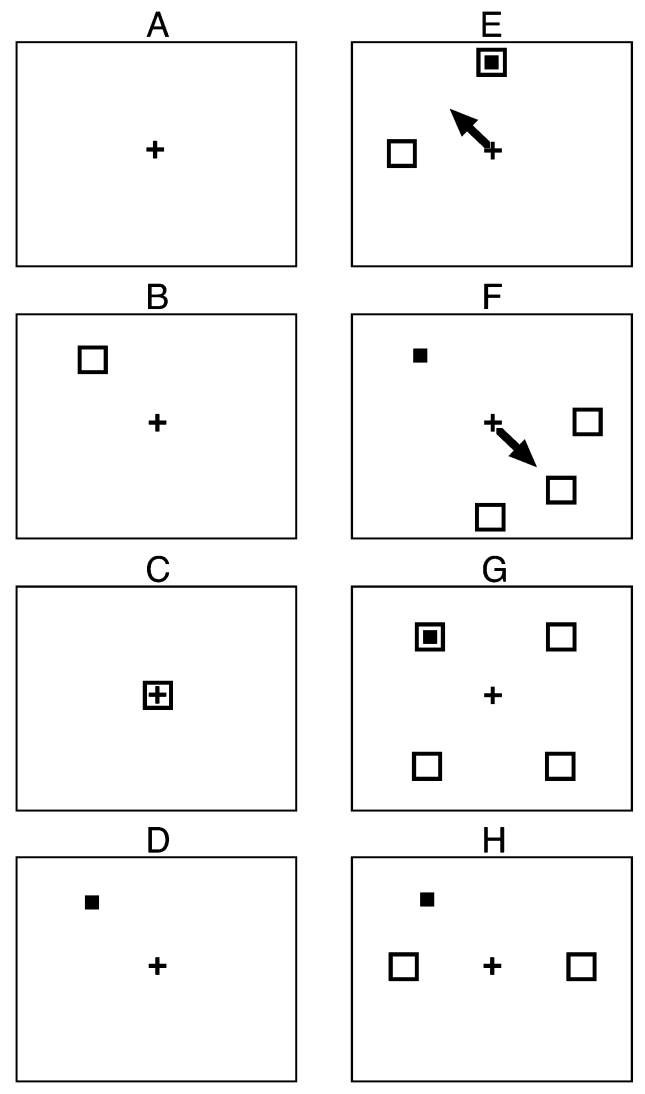

Figure 1. Sample displays, with contrast reversal for convenience. A sample trial sequence is illustrated in panels $A-D$. (A) A fixation cross was the only stimulus present at the start of a trial. (B) A single cue was added to the display for $200 \mathrm{msec}$. (C) When the peripheral cue(s) was (were) removed, a cue to return to fixation was presented for 200 msec. (D) A peripheral target is shown that, for this sequence, would be a cued target. Several cue-target relationships are illustrated in panels $\mathbf{E}-\mathbf{H}$. Cues and targets are shown together, although they never occurred together in the experiment. When the cue has a net vector of $>0$, its direction is shown by the arrow. (E) A double cue shown with a cued target that is $\mathbf{4 5}^{\circ}$ from the net vector of the cue. (F) A triple cue shown with an uncued target that is $180^{\circ}$ from the net vector of the cue. (G) A quadruple cue with a zero net vector is shown with a cued target. (H) A double cue with a zero net vector is shown with an uncued target. 
coming target's location, and was instructed to press a response button as soon as he or she detected the target. To initiate the events of a trial, the participant pressed the space bar with the left index finger while the right index finger remained on the button box. The trial did not begin and the program awaited another space bar response if EyeLink did not detect fixation stability. After successful initiation, a randomly selected one to four of the eight possible cues were presented for $200 \mathrm{msec}$. The cue display was immediately followed by a cue to return to fixation for $200 \mathrm{msec}$. At this point (cue-target stimulus onset asynchrony [SOA] $=400 \mathrm{msec}$ ) or $1,100 \mathrm{msec}$ later (cue-target SOA $=1,500 \mathrm{msec}$ ), the target, which appeared randomly at one of the eight peripheral locations or at fixation, was presented until the response.

\section{Design}

The participants completed four blocks, consisting of 192 trials each. Peripheral targets occurred on two thirds of the trials; central targets occurred on the remaining third. As in Wright and Richard (1996), trials from the 1,500-msec condition, which occurred $25 \%$ of the time, served as catch trials to minimize anticipatory responding on the remaining, experimental trials. Trials with each possible number of cues (one, two, three, and four) occurred equally often in each combination of target location (central/peripheral) and SOA $(400 / 1,500)$. Regardless of the number of locations that were cued, when a peripheral target was presented, it was equally likely to occur at each of the eight possible locations, whether these had been cued or not.

\section{RESULTS}

The first 20 trials in Block 1 and the first 5 trials in each of the remaining blocks were considered practice trials and were discarded from the analysis. The rate of anticipatory responding (buttonpress prior to target appearance) was $2.1 \%$ and $7.9 \%$ on experimental and catch trials, respectively. RT analyses excluded any trials on which the participants failed to maintain fixation during the period from trial initiation until the manual response.
Eye movements were detected on $29.8 \%$ of the experimental trials and on $45.2 \%$ of the catch trials. ${ }^{2}$ Trials with RTs less than $150 \mathrm{msec}$ and greater than $750 \mathrm{msec}$ (in total, fewer than 5\%) were also excluded.

We first examined performance as a function of the angular distance between the target and the average direction of the cue. As can be seen in Figure 2A, RT to peripheral targets declined monotonically as this distance increased $[F(4,80)=5.32, p<.001]$. For comparison with the single-cue data, multicue arrangements with a substantial average vector were combined. The criterion for substantial can be easily conceptualized in terms of the distance between the center of gravity of the cue and fixation, which had to exceed $1.5^{\circ}$ (or $20 \%$ of the distance from fixation to the imaginary circle containing cues and peripheral targets). A significant $[F(4,80)=$ $9.28, p<.0001]$ gradient, whose slope was very similar to that observed following a single cue, was obtained (Figure 2B). The data from the two panels of Figure 2 were subjected to an analysis of variance (ANOVA), which revealed a significant effect of distance $[F(4,80)=$ $11.69, p<.0001]$, no effect of number of cues (one vs. more than one; $F<1)$, and no interaction $(F<1)$.

Several interesting comparisons are made possible ${ }^{3}$ by the random generation of cue arrangements. The multicue data from Figure 2B were sorted according to whether the target had been presented at a previously cued or an uncued location, revealing similar results for these two conditions (Figure 3 ). There was no main effect of cuing $(F<1)$, and the two functions have essentially identical slopes. In other words, when the cue array generated a net vector away from fixation, there was inhibition, independent of stimulation, in the direction of the array's net vector. When there are two or four cues, a net vector of zero amplitude is possible. This occurs when two cues
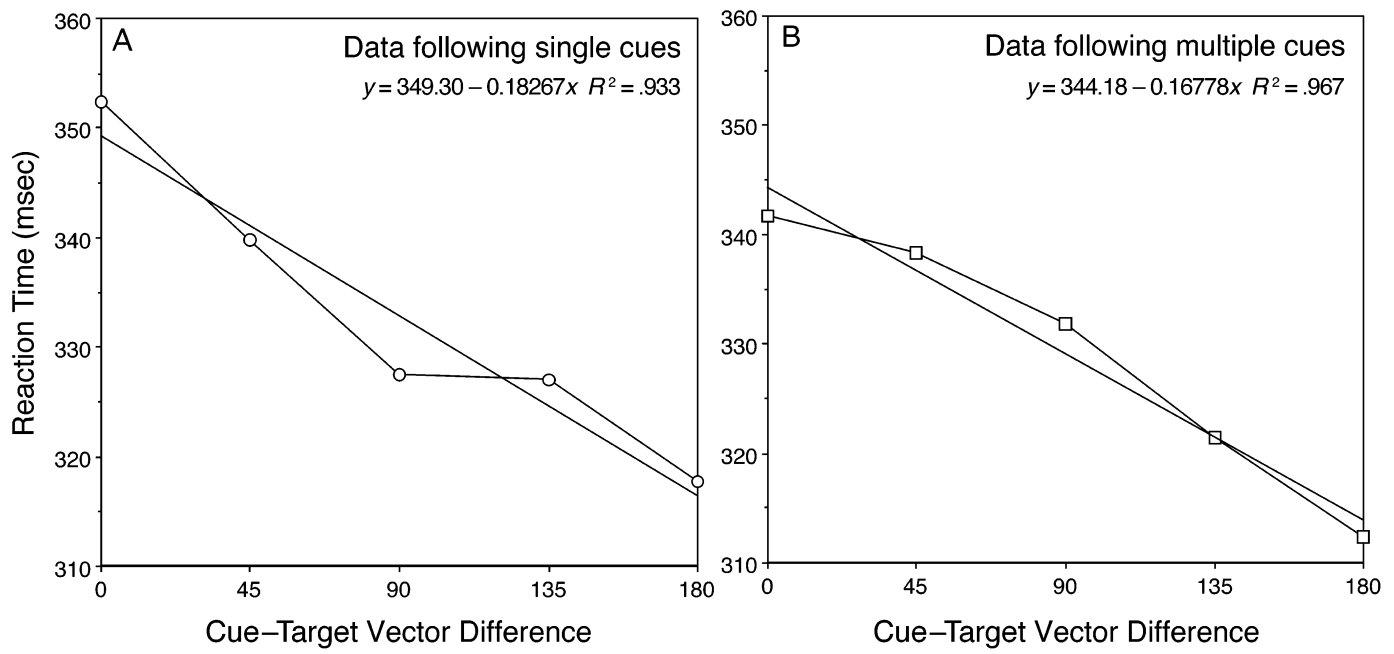

Figure 2. Reaction time as a function of the angular difference between the net vector of the cue and the net vector of the target. (A) Performance following single cues. (B) Performance following multiple cues with a substantial net vector (see the text for an explanation). 


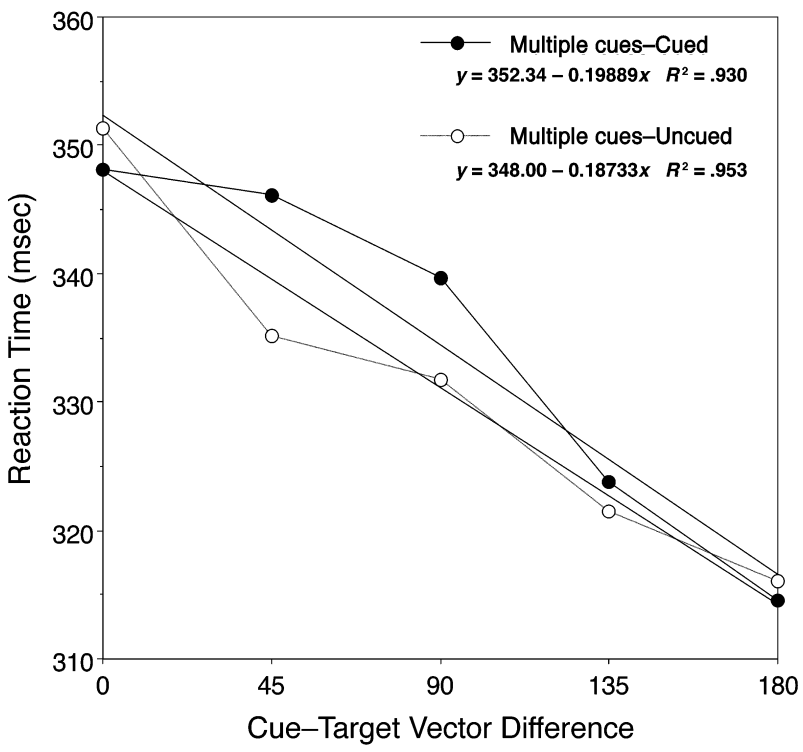

Figure 3. The data from Figure 2B, split by whether the target appeared at a cued or an uncued location. Note that, in this figure, each trial contributing to a condition is weighted equally (see note 3$)$.

are at opposite locations, four cues are at every other location, or four cues occur in pairs at opposite locations (see Figure 1, panels $\mathrm{G}$ and $\mathrm{H}$, for two examples). The data from such trials were sorted according to whether targets appeared at cued and uncued locations. If IOR is coded in relation to the net vector of the cue, then, with these arrangements, there should be no IOR. On the other hand, if IOR is generated by peripheral stimulation, there should be a substantial advantage for the uncued locations. On all such zero net vector multicue trials combined, RT was 330.9 and $334.3 \mathrm{msec}$ for targets at cued locations versus those at uncued locations, respectively (a difference that was not significant; $F<1$ ). Together with the previous finding (Figure 3 ), this absence of IOR provides compelling evidence that, in this experiment, it was the net vector elicited by the peripheral stimuli, rather than the individual elements making up the cue, that was primarily responsible for the pattern of inhibition.

To allow comparison with the findings in Wright and Richard (1996), mean RT was subjected to an ANOVA with number of cues (one to four) and target condition (cued, uncued, or central) as within-subjects factors. Both main effects were significant [number of cues, $F(3,63)=15.273, M S_{\mathrm{e}}=3,800.5, p<.001$; target condition, $\left.F(2,42)=31.344, M S_{\mathrm{e}}=38,366.3, p<.001\right]$, whereas the interaction was not $(F<1)$. RT decreased as the number of cues increased and was shortest to targets presented centrally. As in Wright and Richard (1996), the cued minus uncued RT difference (about $14 \mathrm{msec}$ ) was significant $\left[F(1,21)=9.854, M S_{\mathrm{e}}=8,287.6, p=\right.$ $.005]$ and was unaffected by number of cues $(F<1)$.

\section{DISCUSSION}

When the RT at all the uncued locations was subtracted from that at all the cued locations, the difference was about $14 \mathrm{msec}$ and was relatively unaffected by the number of cued locations. Although this finding is quite similar to that obtained, using the same subtractions, in the study by Wright and Richard (1996), ${ }^{4}$ we discovered a much more interesting and important pattern of results by considering the relation between the net direction of the cue and the direction of the target. This pattern includes four findings, supporting the following two empirical generalizations: (1) There is a directional gradient of inhibition centered on the net direction of the cue, and (2) there is little or no additional local inhibition due to stimulation at a cued location.

First, following a single cue, RT was longest at the cued location and decreased monotonically as the angular distance between the cue and the target increased (see Figure 2$)$. The overall difference between cued $\left(0^{\circ}\right.$ difference between cue and target; i.e., the cue and the target were at the same location) and uncued opposite $\left(180^{\circ}\right.$ from the cue) RTs following a single cue, which would be the measure of IOR in typical 2-location studies, was about $35 \mathrm{msec}$. The gradient we observed following a single cue is consistent with that reported originally by Maylor and Hockey (1985), using a linear array of targets, and by Bennett and Pratt (2001), using 221 target locations. Our circular layout produced a pattern similar to those reported by Pratt et al. (1999, Experiment 3), Snyder, Schmidt, and Kingstone (2001), and Dorris et al. (1999). Second, following multiple simultaneous cues that might elicit activation of a saccade (i.e., whose center of gravity was at least $1.5^{\circ}$ away from fixation), the slope of the gradient as the target's angular distance from the net direction of the cue increased (see Figure 2B) was very similar to that following a single cue.

Third, RTs following multiple cues, although strongly affected by the net direction of the cue, were almost unaffected by whether the target appeared at a location that had been stimulated by a cue element. Indeed, when there was a substantial net vector associated with the cue, the overall difference between targets presented at stimulated and unstimulated locations was a nonsignificant 3-4 msec. Fourth, for cue arrays whose net vector was zero (center of gravity fell on fixation), there was no difference between RTs to cued (330.9-msec) and uncued (334.3-msec) targets.

Our study generated data that can address a longstanding conflict in the literature on IOR. Posner and Cohen (1984) and Maylor (1985) compared performance following single and double (opposite) cues, to determine whether IOR is caused by stimulation or by the kind of asymmetric stimulation that might elicit orienting. Unlike these two studies, which, because there were only two possible target locations, had to compare performance across trials with different types of cues (uncued on single vs. cued on double), the present study has 
the benefit of allowing a comparison of cued and uncued locations following the same cue with a null net vector. ${ }^{5}$ Our finding of no difference between cued and uncued RTs on trials when the net vector was zero reinforces Maylor's pattern of results (cued RT following a double opposite cue was more similar to uncued RT than to cued RT following a single cue), as well as her interpretation that the cause of IOR is more closely linked with exogenous orienting than it is with peripheral stimulation.

Although our findings (see Figures 2 and 3) strongly point toward orienting (e.g., an eye movement or a shift of attention), rather than local stimulation, as the generator of IOR, further studies will be required to determine their generality and to identify the neural mechanism(s) that might underpin them. In this regard, we are encouraged by Tipper's (e.g., Tipper, Howard, \& Houghton, 1998) proposal that population coding by a neural action system will determine the net direction of the action. Although Tipper suggested that more than one task-relevant action may be activated at a time, this idea was put forward for situations in which a target and a distractor were presented simultaneously and, hence, each item had to be individuated so that filtering mechanisms could suppress the action code activated by the distractor while favoring those activated by the target. Our finding, which suggests that the individual cue elements are not activating distinct action codes, is not at all inconsistent with Tipper's suggestion. In our task, the entire cue array is irrelevant and, therefore, not task relevant. We imagine that because our observers are trying to filter out the cue and, hence, all of its elements, they are therefore processed as one irrelevant episode. This leads to one encoded object/ event and, hence, one activated action code. Of course, this is filtered from some levels of processing (e.g., no eye movement is actually made, and participants do not respond to the cue with a buttonpress). Nevertheless, via population coding in neural systems mediating overt or covert orienting, initiated by all of the cue's elements, we believe that an orienting response (saccade and shift of attention) is programmed to the center of gravity (McGowan, Kowler, Sharma, \& Chubb, 1998) or center of area (Melcher $\&$ Kowler, 1999) of the elements making up the cue. To the extent that the direction of the target is in the same direction as this prior orienting response, covert orienting is delayed and, hence, RT to the target is lengthened; and as the target's direction differs from the inhibited direction, RT improves (see Klein \& MacInnes, 1999, and MacInnes \& Klein, 2003, for converging evidence).

\section{REFERENCES}

Bennett, P. J., \& Pratt, J. (2001). The spatial distribution of inhibition of return. Psychological Science, 12, 76-80.

Danziger, S., \& KingSTONE, A. (1999). Unmasking the inhibition of return phenomenon. Perception \& Psychophysics, 61, 1024-1037.

Dorris, M. C., TaYlor, T., Klein, R. M., \& Munoz, D. P. (1999). Influence of previous visual stimulus or saccade on saccadic reaction times in monkey. Journal of Neurophysiology, 81, 2429-2436.

Fernandez-Duque, D., \& PosNeR, M. I. (1997). Relating the mechanisms of orienting and alerting. Neuropsychologia, 35, 477-486.
KLEIN, R. M. (1988). Inhibitory tagging system facilitates visual search. Nature, 334, 430-431.

KLEIN, R. M. (2000). Inhibition of return. Trends in Cognitive Sciences, 4, 138-147.

KLEIN, R. M., \& MACINNES, J. (1999). Inhibition of return is a foraging facilitator in visual search. Psychological Science, 10, 347-352.

Knapp, W. H., III, \& Abrams, R. A. (2001, November). Automatic multilocation inhibition of return. Paper presented at the 42nd Annual Meeting of the Psychonomic Society, Orlando, FL.

MacInNES, J. W., \& KLEIN, R. M. (2003). Inhibition of return biases orienting during the search of complex scenes. Scientific World Journal, 3, 75-86.

MAYLOR, E. A. (1985). Facilitatory and inhibitory components of orienting in visual space. In M. I. Posner \& O. S. M. Marin (Eds.), Attention and performance XI (pp. 189-204). Hillsdale, NJ: Erlbaum.

MAYloR, E. A., \& Hockey, R. (1985). Inhibitory component of externally controlled covert orienting in visual space. Journal of Experimental Psychology: Human Perception \& Performance, 11, 777-787.

McGowan, J. W., Kowler, E., Sharma, A., \& ChubB, C. (1998). Saccadic localization of random dot targets. Vision Research, 38, 895909.

Melcher, D., \& Kowler, E. (1999). Shapes, surfaces and saccades. Vision Research, 39, 2929-2946.

PoSNER, M. I., \& COHEN, Y. (1984). Components of visual orienting. In H. Bouma \& D. G. Bouwhuis (Eds.), Attention and performance $X$ : Control of language processes (pp. 551-556). Hillsdale, NJ: Erlbaum.

Pratt, J., SpaleK, T. M., \& Bradshaw, F. (1999). The time to detect targets at inhibited and noninhibited locations: Preliminary evidence for attentional momentum. Journal of Experimental Psychology: Human Perception \& Performance, 25, 730-746.

RaFal, R. D., Calabresi, P. A., Brennan, C. W., \& Sciolto, T. K. (1989). Saccade preparation inhibits reorienting to recently attended locations. Journal of Experimental Psychology: Human Perception \& Performance, 15, 673-685.

SNYDER, J. J., \& KInGSTONE, A. (2000). Inhibition of return and visual search: How many separate loci are inhibited? Perception \& Psychophysics, 62, 452-458.

SNYDER, J. J., SchmidT, W. C., \& Kingstone, A. (2001). Attentional momentum does not underlie the inhibition of return effect. Journal of Experimental Psychology: Human Perception \& Performance, 27, 1420-1432.

TAYLOR, T. L., \& KLEIN, R. M. (1998). On the causes and effects of inhibition of return. Psychonomic Bulletin \& Review, 5, 625-643.

Tipper S. P., Howard L. A., \& Houghton, G. (1998). Action-based mechanisms of attention. Philosophical Transactions of the Royal Society of London: Series B, 353, 1385-1393.

WRIGHT, R. D., \& RiCHARD, C. M. (1996). Inhibition-of-return at multiple locations in visual space. Canadian Journal of Experimental Psychology, 50, 324-327.

WRIGHT, R. D., \& RICHARD, C. M. (2000). Location cue validity affects inhibition of return of visual processing. Vision Research, 40, 23512358 .

\section{NOTES}

1. This is distinct from the question of whether IOR can be observed at multiple locations when these are cued sequentially. With sequential cues, there is now fairly good evidence that each orienting event leaves an inhibitory tag, with the older tags weakened by the passage of time and the subsequent cues (Knapp \& Abrams, 2001; Snyder \& Kingstone, 2000).

2. The relatively high rate of detected gaze shifts is likely explained by the fact that the threshold for detecting them was low and, when they were detected, no feedback was provided to the participants. To determine the degree to which our findings might depend on the rate of gaze shifts detected, we conducted analyses excluding the 3 participants with the highest rate of gaze shifts and including, as a between-group factor, eye movement propensity based on a mean split. None of the important patterns was undermined by these analyses.

3. Since the choice of cued and targeted locations was entirely random (with the exception of the requirements that there be equal num- 
bers of trials for each number of cues and that the cue array be entirely unpredictive of target location) and different numbers of trials were excluded per participant per condition, there was huge variability across participants in the number of arrangements that fell into the categories used in most of the subsequent analyses, and there were many empty cells contributed by different participants. Hence, in the analyses reported in this paragraph, trial, rather than participant, becomes the random effect, and the mean RTs we report were calculated by summing all the RTs in a particular category and dividing by the number of trials contributing RTs. Analyses were also conducted on normalized RTs ( $z$-score conversion, using each participant's overall mean and standard deviation of RT) in order to remove variance due to individual differences in RT. Since the two kinds of ANOVAs agreed, we will report only the ANOVA on RTs.

4. This replication suggests that perhaps Wright and Richard's (1996) participants may have been unaware of the cue-target contingencies, or the methods used to encourage attention's removal from the periphery
(33\% central targets; cue to return to fixation) may have overcome any tendency to utilize them.

5. Studies of orienting and alerting by Fernandez-Duque and Posner (1997) and by Danziger and Kingstone (1999) also permit such a comparison. Although Fernandez-Duque and Posner's study was not about IOR, at relatively long cue-target intervals where IOR might have been present, there was (as in our study) little difference in RT to targets presented at four simultaneously cued locations $\left(90^{\circ}\right.$ apart) and to targets presented at the four intermediate, uncued locations. In Danziger and Kingstone, cues and targets were presented at locations spaced $90^{\circ}$ apart. Uninformative double cues were used, but the data from those with opposite (zero net vector) cues were not separated from those with adjacent cues.

(Manuscript received March 30, 2004; revision accepted for publication July 22, 2004.) 